February 2, 2008

LBNL-62220

\title{
Field Theory On The World Sheet: Mean Field Expansion And Cutoff Dependence 1
}

\author{
Korkut Bardakci2 \\ Department of Physics \\ University of California at Berkeley \\ and \\ Theoretical Physics Group \\ Lawrence Berkeley National Laboratory \\ University of California \\ Berkeley, California 94720
}

\begin{abstract}
Continuing earlier work, we apply the mean field method to the world sheet representation of a simple field theory. In particular, we study the higher order terms in the mean field expansion, and show that their cutoff dependence can be absorbed into a running coupling constant. The coupling constant runs towards zero in the infrared, and the model tends towards a free string. One cannot fully reach this limit because of infrared problems, however, one can still apply the mean field method to the high energy limit (high mass states) of the string.
\end{abstract}

\footnotetext{
${ }^{1}$ This work was supported in part by the Director, Office of Science, Office of High Energy and Nuclear Physics, of the U.S. Department of Energy under Contract DE-AC02$05 \mathrm{CH} 11231$.

${ }^{2}$ e-mail: kbardakci@lbl.gov
} 


\section{Introduction}

The present work is the continuation of a program developed in a series of earlier papers [1-7]. The main goal of this program was to study the sum of planar graphs of a given field theory by first reformulating it as a two dimensional local theory on the world sheet. This reformulation makes it possible to do dynamical calculations using the mean field approximation. With the help of this approximation, it was possible to show string formation in the case of simplest of field theories, a scalar theory with a cubic interaction, thus building a bridge between field theory and the string theory.

Although we believe that a promising start has been made in addressing an old and important problem [8], there are still many questions left to be answered. To name a few, the world sheet parametrization was based on light cone kinematics, with the resulting loss of manifest Lorentz covariance. The meanfield method has so far not been applied to more realistic theories than the scalar cubic theory. Also, since this method can be formulated as an expansion in inverse powers of the transverse space dimensions, the expansion parameter may not be all that small and its convergence is questionable.

In this article, we will address another problem, which is as important as any listed above. In doing so, we will also indirectly shed light on the problem of convergence of the mean field expansion. Both in constructing the world sheet theory, and also in applying the mean field method, it was necessary to introduce cutoffs. These were taken to be the spacings of the two grids set up on the world sheet, or alternatively the corresponding cutoffs in momentum space. Of course, ultimately one would like to get rid of them by renormalizing the model [9]. However, in this case, renormalization is not a completely straightforward matter. In particular, one is rather limited in the choice of counter terms in the initial action. Nevertheless, it was shown in references [4-7] that, by assigning a natural cutoff dependence to the original parameters of the model, in the leading order of the mean field expansion, the slope of the emerging string came out finite. One can then hope that other physical quantities will also turn out to be cutoff independent.

As is well known, the cutoff dependence of a field theory is intimately related to its scaling properties. The renormalization group equations are in general based on this connection. In the world sheet theory we are studying, there is also a connection between scaling and Lorentz invariance. This is because the world sheet parametrization we are using is based on the light cone variables, with the resulting loss of manifest Lorentz covariance. In this 
picture, the boost along the special light cone direction is implemented by the scaling of the world sheet coordinates. It then follows that scale invariance is a necessary (but not sufficient) condition for Lorentz invariance. We therefore see that the questions of cutoff independence and Lorentz invariance are connected; the necessary (and for cutoff independence, sufficient) condition for both is scale invariance.

This paper is mainly devoted to the study of scaling behaviour and the cutoff dependence of the world sheet model that represents the massless scalar with cubic interaction. The tool we will use will be the mean field expansion, which is an expansion in inverse powers of $D$, the dimension of transverse space. In order to make this paper self contained, in section 2, we will briefly review the derivation of the world sheet model corresponding to the massless scalar field theory with cubic interaction, and in section 3, we will discuss the application, in the leading order, of the mean field method to the model. Within this approximation, we will be able to demonstrate string formation on the world sheet. We will also discuss how the two cutoffs are introduced on the world sheet, and how various fields and parameters transform under scaling, and how this is related to their cutoff dependence. The material covered in these two sections is mostly a review of the earlier results [1-6].

A crucial step in the derivation of the results reviewed here was the minimizing of the ground state energy. Since the ground state energy is cutoff dependent, one may wonder how much a result such as string formation depends on the regulation scheme used. In section 4, we generalize the sharp cutoffs used so far to a smooth cutoff with an two arbitrary profile functions. We show that so long as the profile functions are positive, the result about string formation remains valid. This section, as well as the rest of the article, contains material that is mostly new.

The previous sections only dealt with the leading order in the expansion in powers of $1 / D$. The higher order terms in this expansion can be computed in two steps: In the first step, we compute an effective action by integrating over the field q, the world sheet field that represents the transverse momenta. In section 5, we show that this effective action can be written as a generalized sigma model in a new dynamical field $\phi$. We then expand this action simultaneously in powers of $1 / D$ and in the number of derivatives with respect to the world sheet coordinates, and we determine explicitly the leading term of this expansion, which provides the potential energy for $\phi$, as well as the next term, which provides the kinetic energy. These terms were already derived in references $[5,6]$. The field $\phi$ plays an important role in the model; for 
example, a non-vanishing ground state expectation value for $\phi$ generates a non-vanishing string slope. We also investigate the cutoff dependence of the action, and show that, up to logarithmic corrections, the dependence on the powers of cutoff is what is expected on the basis of simple power counting.

The next step of the program is to use this sigma model as the starting point for computing higher order contributions. Here, however, we face the unusual situation of having an initial action that is cutoff dependent. Of course, higher order contributions will generate further cutoff dependence. This brings up the question, taken up in section 6 , of how to renormalize the model, so that observables become cutoff independent. In standard field theory, divergences are normally cancelled by introducing counter terms in the original action. Here, since $\phi$ started life as an auxilliary field in the original action, we do not have the freedom to introduce arbitrary counter terms. Before trying to answer this question, we can ask even a more basic one: What are the observables? It is natural to assume that in a theory in flat space, all observable quantities can ultimately be expressed in terms of Lorentz invariant ones. In a covariantly formulated theory, this is a trivial requirement, but here, the situation is different, since it is not at all straightforward to identify Lorentz invariant objects. Since Lorentz invariance implies scale invariance, we impose on the observables the more modest requirement of scale invariance. Again, by simple power counting, scale invariant objects are shown to have no power dependence on the cutoff. Consequently, Lorentz invariance means no dependence on powers of the cutoff.

However, there still remains a logarithmic dependence on the cutoff, to which we turn next. By means of a simultaneous scaling of both the coordinates and the field $\phi$, we transform the action into a new form, which no longer has power dependence on the cutoff. In the new action, the original expansion parameter is replaced by $\lambda^{2}$, which is inversely proportional to the log of the cutoff. Also, terms with more than two derivatives are suppressed by inverse powers of the same log of the cutoff. We note that, depending on the cutoff, $\lambda^{2}$ could be smaller than $1 / D$ and thus provide a better parameter of expansion.

In section 7 , we consider the perturbation expansion based on the action above and on an expansion in powers of $\lambda$. We show that the higher order contributions do not change the cutoff structure of the initial action in any substantial way. The logarithmic dependence of both $\lambda$ and of the terms with more than two derivatives remain unmodified.

We conclude section 7 with a discussion of these results from the point of 
view of the Wilsonian renormalization scheme. Starting with an initial cutoff $\Lambda$, we try to reduce it to a much lower value $\mu$, by continually integrating over the high end of the spectrum. As we do this, the coupling constant, which is inversely proportional to $\ln (\Lambda / \mu)$, runs towards zero, and the model tends towards a free string. As expected, this limit, the infrared fixed point of the model, is scale invariant and cutoff independent. However, this result rests on various approximations, which are valid only in the ultraviolet regime. Consequently, it is not possible to go all the way to the infrared and reach this fixed point. We argue, however, that by keeping $\mu$ large enough, we can probe the asymptotic spectrum of the string, but not the lower lying states. The last section summarizes our conclusions.

\section{The World Sheet Action}

This and the next section are devoted to a review of the results obtained in Refs.[1-6]. The basic idea is first to represent the sum of planar graphs of a given field theory as a local field theory on the world sheet, and then use the mean field approximation to show string formation. Starting with a world sheet parametrized by the light cone variables

$$
\tau=x^{+}=\left(x^{0}+x^{1}\right) / \sqrt{2}, \quad \sigma=p^{+}=\left(p^{0}+p^{1}\right) / \sqrt{2},
$$

a general planar Feynman graph for the massless scalar theory with cubic interaction in $D+2$ dimensions can be represented by a bunch of solid lines (Fig.1)[10]. One associates a particular transverse momentum $\mathbf{q}_{n}$ with the n'th line, and two adjacent lines $n$ and $n+1$, with momenta $\mathbf{q}_{n}$ and $\mathbf{q}_{n+1}$,

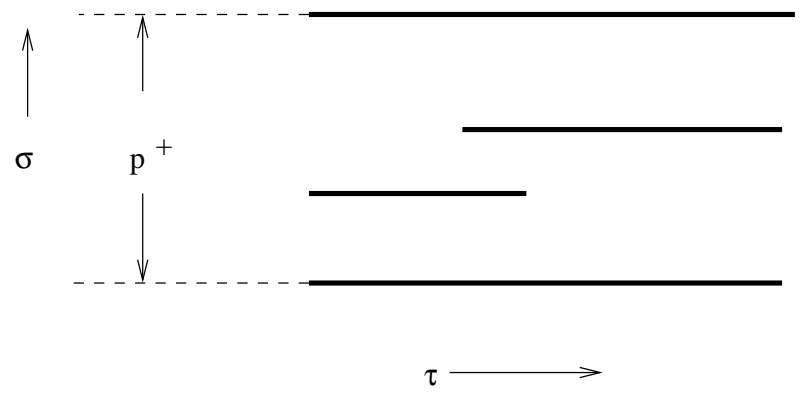

Figure 1: A Typical Graph 
represent the light cone propagator

$$
\Delta(p)=\frac{\theta(\tau)}{2 p^{+}} \exp \left(-i \tau \frac{\mathbf{p}^{2}}{2 p^{+}}\right),
$$

where $\mathbf{p}=\mathbf{q}_{n}-\mathbf{q}_{n+1}$. A factor of $g$ is inserted at the beginning and at the end of each line, where the interaction takes place.

The light cone Feynman rules sketched above can be reproduced by a local field theory on the world sheet. Here, we summarize the results, and refer the reader to [1-6] for the detailed derivations. The transverse momenta $\mathbf{q}$, originally defined only on the solid lines, can be promoted to a local field $\mathbf{q}(\sigma, \tau)$ over the whole world sheet. If we think of the solid lines as boundaries on the world sheet, and the rest as the bulk, $\mathbf{q}(\sigma, \tau)$ satisfies the equation

$$
\partial_{\sigma}^{2} \mathbf{q}(\sigma, \tau)=0
$$

in the bulk, and the Dirichlet boundary condition

$$
\partial_{\tau} \mathbf{q}(\sigma, \tau)=0
$$

on the solid lines, since the $\mathbf{q}$ flowing through a solid line is constant ( $\tau$ independent). With the help of a Lagrange multiplier $\mathbf{y}(\sigma, \tau)$, both the equations of motion and the boundary conditions are incorporated into the following action:

$$
S_{q}=\int_{0}^{p^{+}} d \sigma \int d \tau\left(-\frac{1}{2} \mathbf{q}^{\prime 2}+\rho \mathbf{y} \cdot \dot{\mathbf{q}}\right)
$$

where a dot represents the derivative with respect to $\tau$ and a prime the derivative with respect to $\sigma$. The function $\rho$ is defined to be the sum of Dirac delta functions with support on the solid lines.

In this expression for $S_{q}$, one has to integrate functionally not only over $\mathbf{q}$ and $\mathbf{y}$, but also over the positions and the lengths of the solid lines. This is best accomplished by introducing a two component fermion $\psi_{i}(\sigma, \tau), i=1,2$, and its adjoint $\bar{\psi}_{i}$, and setting

$$
\rho=\frac{1}{2} \bar{\psi}\left(1-\sigma_{3}\right) \psi
$$

The action for the fermions is

$$
S_{f}=\int_{0}^{p^{+}} d \sigma \int d \tau\left(i \bar{\psi} \dot{\psi}-D g \bar{\psi} \sigma_{1} \psi\right)
$$




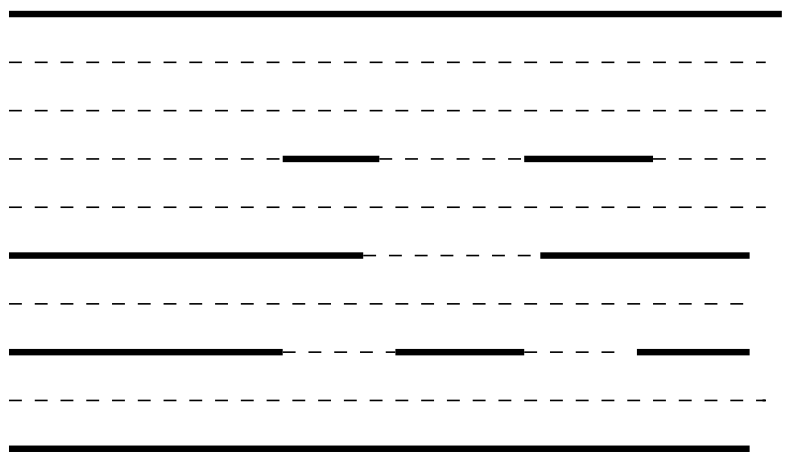

Figure 2: Solid And Dotted Lines

To see clearly how this works and to avoid singular expressions, it is best to discretize the sigma coordinate into segments of length $a$. This discretization is pictured in Fig.2 as a collection of parallel line segments, some solid and some dotted, spaced distance $a$ apart. The boundaries are marked by the solid lines, associated with the $i=2$ component of the fermion, and the bulk is filled by the dotted lines, associated with the $i=1$ component. The first term in Eq.(6) represents the free propagation of the fermion, tracing a solid or a dotted line, and the second term, which converts the dotted line into a solid line or vice versa, represents the interaction. We have scaled the coupling constant $g$ by the dimension of the transverse space $D$ in anticipation of the large $D$ limit, discussed in the next section. Finally, integrating over the fermion field is then the same as summing over the location and the length of the boundaries.

There is one more ingredient needed to complete the world sheet action. In Eq.(4), the part of the integral over $\mathbf{y}$ that has support in the bulk (dotted lines) diverges, since the integrand is $\mathbf{y}$ independent in this region. To avoid this problem, we add a Gaussian term to action which cuts off the divergence:

$$
S_{g . f}=\int_{0}^{p^{+}} d \sigma \int d \tau\left(-\frac{1}{2} \alpha^{2} \bar{\rho} \mathbf{y}^{2}\right) .
$$

Here $\alpha$ is a fixed parameter, of which we will say more later on, and $\bar{\rho}$ is defined by

$$
\bar{\rho}=\frac{1}{2} \bar{\psi}\left(1+\sigma_{3}\right) \psi
$$

and it is complementary to $\rho$ : It vanishes on the solid lines and has support only in the bulk. In fact, on the world sheet regulated by the grid, one can 
set

$$
\rho+\bar{\rho}=\bar{\psi} \psi \rightarrow 1 / a
$$

Finally, the complete world sheet action is given by

$$
\begin{aligned}
S & =S_{q}+S_{f}+S_{g . f} \\
& =\int_{0}^{p^{+}} d \sigma \int d \tau\left(-\frac{1}{2} \mathbf{q}^{\prime 2}+\rho \mathbf{y} \cdot \dot{\mathbf{q}}-\frac{1}{2} \alpha^{2} \bar{\rho} \mathbf{y}^{2}+i \bar{\psi} \dot{\psi}-D g \bar{\psi} \sigma_{1} \psi\right)
\end{aligned}
$$

An important symmetry any light cone action must satisfy follows from invariance under the boost along the special direction we have labeled as 1 . This boost translates into the scaling of the world sheet coordinates by

$$
x^{+} \rightarrow u x^{+}, p^{+} \rightarrow u p^{+},
$$

and under which the fields transform as

$$
\begin{aligned}
& \mathbf{q}(\sigma, \tau) \rightarrow \mathbf{q}(u \sigma, u \tau), \quad \mathbf{y}(\sigma, \tau) \rightarrow \mathbf{y}(u \sigma, u \tau) \\
& \psi(\sigma, \tau) \rightarrow \sqrt{u} \psi(u \sigma, u \tau), \bar{\psi}(\sigma, \tau) \rightarrow \sqrt{u} \bar{\psi}(u \sigma, u \tau)
\end{aligned}
$$

The world sheet action given above is not invariant under this scaling, unless the constants $g$ and $\alpha^{2}$ are also scaled according to

$$
g \rightarrow u g, \quad \alpha^{2} \rightarrow u \alpha^{2}
$$

Scale invariance is also violated by the two cutoff parameters needed to regulate the model. We have already introduced one of them, $a$, the grid spacing in the $\sigma$ direction; in order to regulate the integral over $\mathbf{q}$, we will need also to discretize the coordinate $\tau$, with a grid spacing $a^{\prime}$. To preserve scale invariance, at least formally, these two cutoff parameters must also scale like the coordinates:

$$
a \rightarrow u a, \quad a^{\prime} \rightarrow u a^{\prime}
$$

All these definitions are somewhat formal, and there is real danger of violation of scale and hence of Lorentz invariance. We will see later that, at least within the framework of the meanfield approximation, physical quantities, which are by definition cutoff independent, are also scale invariant. Although this is not a complete proof, it provides support for the Lorentz invariance of the whole approach. We will therefore start with the basic assumption that all the scale invariant quantities formed out of the parameters 
$\alpha, g, a$ and $a^{\prime}$ are finite (cutoff independent), so that there is really only one independent cutoff, $a$ or $a^{\prime}$, with the scale invariant ratio

$$
a / a^{\prime}=m^{2}
$$

fixed at a finite value. We also note that $m$, which has the dimensions of mass, is the only dimensionful scale invariant parameter in the problem. It is therefore a natural candidate for a Lorentz invariant mass parameter. We will see later that the slope of the dynamically generated string is proportional to $1 / m^{2}$.

From the remaining parameters $g$ and $\alpha$, we form two new scale invariant parameters by

$$
\bar{\alpha}^{2}=\frac{a^{\prime 2}}{a} \alpha^{2}, \quad \bar{g}=a^{\prime} g
$$

For convenience, we have also made them dimensionless, which can always be achieved by multiplying by appropriate powers of $m$. As the cutoff parameters $a$ and $a^{\prime}$ go to zero, in addition to $m$, we will keep $\bar{g}$ and $\bar{\alpha}$ fixed at a finite values. If we can then show that physical quantities can be expressed solely in terms of these three parameters, their finiteness and scale invariance will follow.

Before closing this section, we would like to note that in addition to the coupling constant $\bar{g}$, an extra parameter $\bar{\alpha}$ appeared. By paying attention to the integration measure in the version of the model with discretized coordinates, it is possible to argue that this parameter should be fixed at

$$
\bar{\alpha}^{2}=\frac{1}{4 \pi} .
$$

However, since the exact value of $\bar{\alpha}$ will not of any importance in what follows, we will leave it undetermined.

\section{The Meanfield Expansion}

The idea behind the meanfield approximation is to recognize that Eq.(10) is the action for a vector model, which can be solved in the large $D$ limit. One first introduces new fields for the scalar products such as $\mathbf{y} \cdot \dot{\mathbf{q}}$ and $\mathbf{y}^{2}$ in order to cast the action into an expression proportional to $D$, the dimension of the transverse space, and then the large $D$ limit is taken by the saddle point method [11]. Here, we choose instead an equivalent but simpler approach. 
Let us add the following term to $S$ of Eq.(10):

$$
\Delta S=\int_{0}^{p^{+}} d \sigma \int d \tau\left(D \kappa\left(\frac{1}{2} \bar{\psi}\left(1-\sigma_{3}\right) \psi-\rho\right)\right)
$$

This term merely enforces the definition of $\rho$ through the Lagrange multiplier $\kappa$. We will use the meanfield method to compute the ground state expectation value

$$
\rho_{0}=\langle\rho\rangle
$$

of $\rho$. It was argued in references [2-7] that a non-zero value for $\rho_{0}$ signals a new phase of the underlying field theory, where the solid lines (boundaries) form a condensate on the world sheet. In contrast, the original perturbative phase corresponds to $\rho_{0}=0$.

The meanfield calculation is simplified by choosing a configuration of the world sheet with the $\sigma$ coordinate compactified with periodic boundary conditions at $\sigma=0$ and $\sigma=p^{+}$, and the total transverse momentum $\mathbf{p}$ set equal to zero. This configuration has the advantage of being translation invariant in both $\sigma$ and $\tau$ directions, so that both $\rho_{0}$ and

$$
\kappa_{0}=\langle\kappa\rangle
$$

can be taken to be constants independent of $\sigma, \tau$. Replacing $\rho, \kappa$ by their constant expectation values in Eq.(10), the integration over $\mathbf{y}$ is easily done, with the result,

$$
\begin{aligned}
S+\Delta S & \rightarrow \tilde{S}_{q}+S^{\prime}+\tilde{S}_{f} \\
\tilde{S}_{q} & =\int_{0}^{p^{+}} d \sigma \int d \tau\left(-\frac{1}{2} \mathbf{q}^{\prime 2}+\frac{1}{2} \beta^{2} \dot{\mathbf{q}}^{2}\right), \\
\tilde{S}^{\prime} & =\int_{0}^{p^{+}} d \sigma \int d \tau\left(-D \kappa_{0} \rho_{0}\right), \\
\tilde{S}_{f} & =\int_{0}^{p^{+}} d \sigma \int d \tau\left(\bar{\psi}\left(i \partial_{\tau}-D g \sigma_{1}+\frac{1}{2} D \kappa_{0}\left(1-\sigma_{3}\right)\right) \psi\right),
\end{aligned}
$$

where

$$
\beta^{2}=\frac{\rho_{0}^{2}}{\alpha^{2} \bar{\rho}_{0}} .
$$

It is clear that a non-zero value for $\rho_{0}$ results in a non-zero string slope $\alpha^{\prime}$ :

$$
\alpha^{\prime 2}=\beta^{2} / \pi^{2}
$$


provided that, as we shall later show,

$$
\bar{\rho}_{0}=\frac{1}{a}-\rho_{0}
$$

is positive, which is necessary for this equation to make sense. This simple argument shows that, independent of any approximation scheme, a non-zero value for $\rho_{0}$ leads to string formation. Whether $\rho_{0}$ is non-vanishing is a question of dynamics. We will compute below the energy of the ground state of the system, using the meanfield approximation, and we will find that it is minimized by a non-vanishing value of $\rho_{0}$. The energy of the ground state $E_{0}$, which follows from the action given by Eq.(17), consists of three pieces:

$$
E_{0}=E_{q}+E^{\prime}+E_{f}
$$

where $E_{q}, E^{\prime}$ and $E_{f}$ are the contributions of $\tilde{S}_{q}, \tilde{S}^{\prime}$ and $\tilde{S}_{f}$ respectively. Since $\kappa_{0}$ and $\rho_{0}$ are constants,

$$
E^{\prime}=D p^{+} \kappa_{0} \rho_{0}
$$

It is also easy to compute $E_{f}$. After discretizing $\sigma$ in steps of length $a$, the system represented by $\tilde{S}_{q}$ consists of $N=p^{+} / a$ number of decoupled one dimensional Ising models. Diagonalizing the resulting two by two matrices, we have

$$
E_{f}^{ \pm}=\frac{D p^{+}}{2 a}\left(\kappa_{0} \pm \sqrt{\kappa_{0}^{2}+4 g^{2}}\right)
$$

Clearly, the ground state energy is minimized by taking the minus sign in front of the square root, so we will drop the plus sign from now on.

Now consider $E_{q}$. We shall only need the leading singular (cutoff dependent) part of $E_{q}$, in the limit $a, a^{\prime} \rightarrow 0$, with $a / a^{\prime}=m^{2}$ kept fixed and finite. In the next section, we will show that, in this limit

$$
E_{q} \rightarrow \frac{D p^{+}}{a^{\prime 2}} F\left(\beta^{2}, m^{2}\right)
$$

where $\beta$, (Eq.(18)), is cutoff independent (finite). It is important to establish the finiteness of $\beta$, since the string slope $\alpha^{\prime}$, which we expect to be finite, is proportional to $\beta$. We could argue this indirectly by observing that $\beta$ is scale invariant; however, it is worthwhile to carry out an explicit calculation. We 
first note that, from its definition on a grid with spacing $a, \rho_{0}$ has a cutoff dependence of the form $1 / a$. It is therefore natural to define

$$
\rho_{0}=x / a, \quad \bar{\rho}_{0}=(1-x) / a .
$$

Going back to $S_{f}$ (Eq.(6)), it is easy to show that $x$ represents the average probability of finding a spin down fermion on the world sheet, which is also the probability of finding a solid line. Therefore,

$$
0 \leq x \leq 1
$$

Expressed in terms of $x$ and $\bar{\alpha}$ (Eqs.(18) and (24)), $\beta$ now reads

$$
\beta=\frac{x}{\bar{\alpha} m^{2} \sqrt{(1-x)}} .
$$

Since the parameters $\bar{\alpha}, x$ and $m$ are all finite as $a, a^{\prime} \rightarrow 0$, it follows that $\beta$ is finite, assuming that $x \neq 1$. From Eq.(19), the string slope also comes out to be finite. This provides an after the fact justification for taking $m^{2}$, the ratio of two cutoffs, to be finite.

Putting together eq.(17),(22) and (23), we have the following expression for the ground state energy:

$$
E_{0}=D p^{+}\left(\frac{1}{a^{\prime 2}} F\left(\beta^{2}, m^{2}\right)-\frac{1}{a} x \kappa_{0}+\frac{1}{2 a}\left(\kappa_{0}-\sqrt{\kappa_{0}^{2}+4 g^{2}}\right)\right)
$$

and the ground state is determined by the saddle point equations

$$
\frac{\partial E_{0}}{\partial \kappa_{0}}=0, \quad \frac{\partial E_{0}}{\partial x}=0 .
$$

The first equation gives

$$
x=\frac{1}{2}\left(1-\frac{\kappa_{0}}{\sqrt{\kappa_{0}^{2}+4 g^{2}}}\right),
$$

and eliminating $\kappa_{0}$ in favor of $x$ from this equation, the ground state energy can be rewritten solely in terms of $x$ ( $\beta$ is a function of $x$ from Eq.(25)):

$$
E_{0}=\frac{D p^{+}}{a^{2}}\left(F\left(\beta^{2}, m^{2}\right)-\frac{1}{m^{2}} \sqrt{x-x^{2}}\right) .
$$


To determine $x$ from the second equation in (27), we need to know the explicit form of $F$. As might be expected, $F$ depends on the cutoff scheme used. We will compute $F$ in the next section, using the cutoff scheme we have introduced, but we will also consider a large class of related methods of regulation. The idea is to see to what extent the results derived here depend on the details of the cutoff scheme. We will show that the ground state energy given by Eq.(29) above has always a minimum at some value of $x=x_{m} \neq 0$, for a large class of cutoff schemes. However, the precise value of $x_{m}$ depends on the scheme used. We therefore conclude that $x_{m} \neq 0$ is a robust result, and string formation with non-zero slope takes place. On the other hand, the parameter $\beta$ (Eq.(25)) and therefore the string slope depends on $x_{m}$. This should not really cause concern, since we can argue as follows: We take $\alpha^{\prime}$ or $\beta$ as a given physical parameter, and for any given value of $x_{m}$, adjust the combination

$$
\bar{\alpha} m^{2}
$$

so as to satisfy Eq.(25). From this point of view, which is the standard idea behind renormalization, $\bar{\alpha}, m^{2}$ and $x_{m}$ are all parameters that depend on the cutoff scheme in use; only the string slope is scheme independent.

\section{Cutoff Dependence Of The Ground State Energy}

In this section, we will investigate the cutoff dependence of the ground state energy, first using two sharp cutoffs, and then replacing these by two smooth profile functions. In particular, we want to see whether, independent of the cutoff scheme used, the potential has a minimum at a non vanishing value of $x$. Integrating over $\mathbf{q}$ in $\tilde{S}_{q}$ (Eq.(17)) gives

$$
\tilde{S}_{q} \rightarrow \frac{1}{2} i D T r \ln \left(\beta^{2} \partial_{\tau}^{2}-\partial_{\sigma}^{2}\right)
$$

and the corresponding energy is

$$
E_{q}=\frac{D p^{+}}{2(2 \pi)^{2}} \int_{-\Lambda_{0}}^{\Lambda_{0}} d k_{0} \int_{-\Lambda_{1}}^{\Lambda_{1}} d k_{1} \ln \left(\beta^{2} k_{0}^{2}+k_{1}^{2}\right) .
$$

Here, we have written the expression for the energy in momentum space, and we have replaced the grid sizes $a^{\prime}$ and $a$ by the equivalent momentum space cutoffs

$$
\Lambda_{0}=\pi / a^{\prime}, \quad \Lambda_{1}=\pi / a .
$$


Also, strictly speaking, instead of an integral over $k_{1}$, we should have a discrete sum in steps of $\Delta k_{1}=2 \pi / p^{+}$. However, since we are only interested in the leading cutoff dependence of $E_{q}$, we can safely replace this sum by an integral.

As explained earlier, we would like to generalize the sharp cutoff used above. Accordingly, we replace Eq.(31) by

$$
E_{q} \rightarrow \frac{D p^{+}}{2(2 \pi)^{2}} \int d k_{0} \int d k_{1} f_{0}\left(k_{0} / \Lambda_{0}\right) f_{1}\left(k_{1} / \Lambda_{1}\right) \ln \left(\beta^{2} k_{0}^{2}+k_{1}^{2}\right)
$$

where the profile functions satisfy the following conditions:

a) $f_{0}(y)=f_{1}(y)=1$ for $-1 \leq y \leq 1$.

b) Both $f_{0}(y)$ and $f_{1}(y)$ are positive functions that rapidly go to zero as $|y| \rightarrow \infty$. Otherwise, they are arbitrary.

Let us now extract $F$ from $E_{q}$ according to Eq.(23), by considering the limit $\Lambda_{0,1} \rightarrow \infty$, with $\Lambda_{0} / \Lambda_{1}=m^{2}$ fixed. The result is

$$
F=\frac{1}{8 m^{2}} \int d y \int d z f_{0}(y) f_{1}(z)\left(\ln \left(m^{2} \beta^{2} y^{2}+z^{2}\right)+\ln \left(\Lambda_{1}\right)\right) .
$$

The term proportional to $\ln \left(\Lambda_{1}\right)$ on the right hand side is independent of $x$ $(\beta)$ and therefore it does not contribute to the equations (27) that determine the ground state. In fact, it is an irrelevant constant which can be subtracted from the ground state energy and we will drop it from now on.

To prove that $E_{0}$ has a minimum at $x_{m} \neq 0$, we have to first show that $F$ is an increasing function of $x$. From the above equation, it is clear that

$$
\partial F / \partial \beta>0
$$

and from Eq.(25),

$$
\partial \beta / \partial x>0
$$

and therefore

$$
\partial F / \partial x>0
$$

For small enough $x$, we can approximate $F$ by

$$
F \approx C_{0}+C_{1} x
$$

where the constant

$$
C_{1}=(\partial F / \partial x)_{x=0}
$$


is positive. On the other hand, the second term in Eq.(29) for $E_{0}$ is negative, and for small $x$, it can be approximated by

$$
-\sqrt{x-x^{2}} / m^{2} \approx-\sqrt{x} / m^{2} .
$$

Comparing this with (35), we see that for small enough $x$, the second term dominates, and $E_{0}$ is a decreasing function of $x$. On the other hand, for $x>$ $1 / 2$, the second term now becomes an increasing function, and consequently, so does $E_{0}$. Since $E_{0}$ is decreasing for small $x$ and starts increasing at $x=1 / 2$, it must have a minimum for some $x_{m}$ in the interval $0<x \leq 1 / 2$.

We note that the only property of $f_{0,1}$ needed in the above argument is their positivity. Therefore, we believe that the result is quite general, and that it holds in any regulator scheme which does not violate the positivity properties of the underlying field theory. Hence, string formation is a robust result, which does not depend on the details of the regulation scheme used. Although the existence of $x_{m} \neq 0$ is thus assured, its value clearly depends on the cutoff functions $f_{0,1}$. We have argued at the end of the last section that $x_{m}, \bar{\alpha}$ and $m^{2}$ are all scheme dependent parameters; only the physical parameter built out of them, $\alpha^{\prime}$, need be scheme independent.

Before closing this section, we would like to mention that the regulation scheme used in reference [3-7] corresponds to taking the small $x$ and therefore the small $\beta$ limit in Eq.(33), which gives the simple result,

$$
F \approx \frac{x}{4 \pi \bar{\alpha} m^{2}} \Lambda_{0}^{2}
$$

\section{Sigma Model For $\phi$}

In this section, we will study higher order corrections to the leading mean field approximation. This will involve evaluating $\tilde{S}_{q}$ (Eq.(30)), not just for a constant $\beta$, as was done in the last section, but with $\beta$ taken to be an arbitrary function of the coordinates. Of course, this cannot be done exactly, so we resort to a double power series expansion described below. Actually, we will explicitly evaluate only the first two terms of this expansion. As for the rest of the terms, we will only be interested in their dependence on the cutoff. We first rewrite Eq.(30) as

$$
\tilde{S}_{q} \rightarrow \frac{1}{2} D \operatorname{Tr} \ln \left(-\partial_{\tau} \phi \partial_{\tau}-\partial_{\sigma}^{2}\right),
$$


where we have renamed $\beta^{2}$ as $\phi(\sigma, \tau)$, and switched to Euclidean metric on the world sheet. From now on, the world sheet action will be written using the Euclidean metric. Next, we expand $\tilde{S}_{q}$ in powers of derivatives with respect to the world sheet coordinates acting on $\phi$ :

$$
\tilde{S}_{q}=\int_{0}^{p^{+}} d \sigma \int d \tau\left(U_{q}(\phi)+\sum_{s=1}^{\infty} Z_{q}^{(s)}(\phi) \partial^{2 s} \phi\right) .
$$

To simplify the notation, we have written the above expansion in a somewhat schematic form. The derivatives are with respect to $\sigma$ or $\tau$, and they could be acting on several $\phi$ 's instead a single one as shown. Therefore, in reality, several terms are collectively represented by a single $Z_{q}^{(s)}$. The common feature of these terms is that they go with $2 s$ number of derivatives, which is the only feature relevant in the following power counting arguments.

This well known expansion is frequently used in the effective potential computations. In conjunction with the loop expansion, it makes the calculation of at least the leading terms in the expansion possible [10]. From our point of view, its chief advantage is that it keeps track of the cutoff dependence of the action. By naive power counting, one would expect each derivative to go with an inverse power of the cutoff. This is true, of course, up to factors involving powers of the logarithm of the cutoff. These logarithmic factors, which will be the focus of our attention, will play an important role later on.

In order to make progress, we need a second expansion, this time in powers of $1 / D$. This is the same $1 / D$ expansion on which the mean field method is based, and it is accomplished by first splitting $\phi$ into two parts as

$$
\phi=\phi_{0}+\chi
$$

and then expanding the trace in Eq.(36) in powers of $\chi$ :

$$
\operatorname{Tr} \ln \left(-\partial_{\tau} \phi \partial_{\tau}-\partial_{\sigma}^{2}\right)=\sum_{0}^{\infty} \frac{(-1)^{n}}{n+1} \operatorname{Tr}\left(-W\left(\Delta^{-1} W\right)^{n}\right)
$$

where,

$$
\Delta=\phi_{0} \partial_{\tau}^{2}+\partial_{\sigma}^{2}, \quad W=\partial_{\tau} \chi \partial_{\tau} .
$$

For convenience of calculation, $\phi_{0}$ is taken to be a constant ( $\sigma, \tau$ independent) background field. We could set it equal to the ground state expectation value of $\phi$; however, we will keep it arbitrary for the time being. The general term 


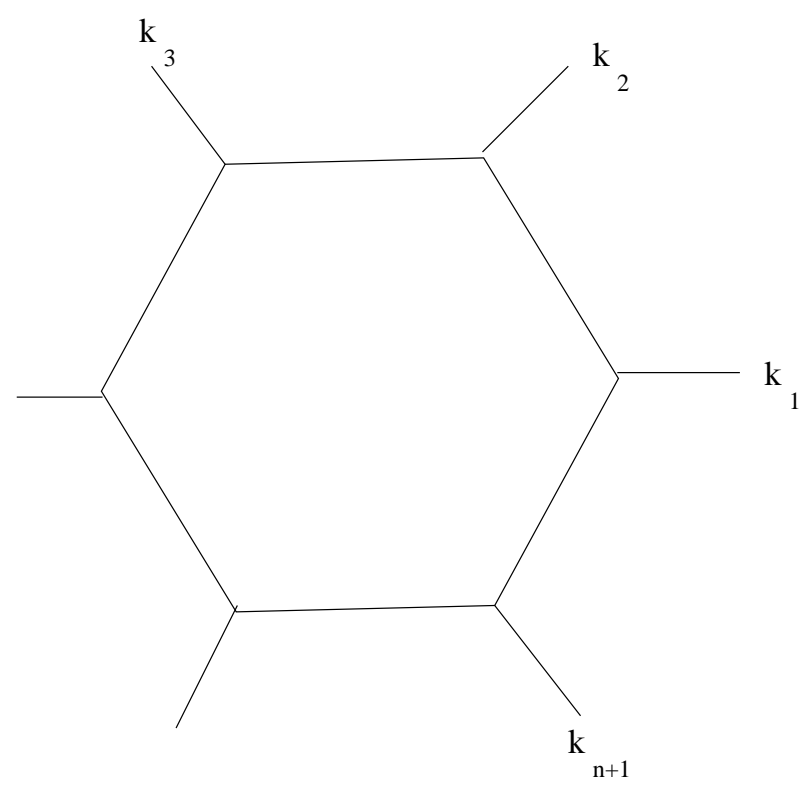

Figure 3: One Loop Graph

in the expansion is the one loop graph with $n+1$ external lines shown in Fig.3.

The next step is to translate the derivative expansion into the momentum space, by expanding the graph in Fig.3 in powers of $k_{1}, k_{2}, \cdots, k_{n+1}$ around the point $k_{1}=k_{2}=\cdots=k_{n+1}=0$. Here, the $k$ 's are two dimensional world sheet momenta carried by the external lines in Fig.3. The term proportional to $Z_{q}^{(s)}$ in Eq.(37) corresponds to a term with the total power $2 s$ in $k$ 's in this expansion. Let us start with the first term of this expansion where all the momenta are set equal to zero; this corresponds to $U_{q}$ in Eq.(37). Setting all the momenta equal to zero is the same as replacing the $n$ 'th power of $\chi$ in the expansion (38) by

$$
\int_{0}^{p^{+}} d \sigma \int d \tau\left(\chi(\sigma, \tau)^{n}\right) .
$$

This leads to the following recipe: Compute

$$
\frac{1}{2} \operatorname{Tr} \ln \left(-\phi_{0} \partial_{\tau}^{2}-\partial_{\sigma}^{2}\right)
$$

for a constant $\phi_{0}$, and in the result replace $\phi_{0}$ by $\phi(\sigma, \tau)$ and then integrate over $\sigma, \tau$. The calculation with a constant background was already done in 
the last section, with $\beta^{2}$ replacing $\phi_{0}$. The result can be expressed in terms of $F$ (Eq.(33)):

$$
U_{q}(\phi(\sigma, \tau))=\frac{D \Lambda_{0}^{2}}{\pi^{2}} F\left(\phi(\sigma, \tau), m^{2}\right) .
$$

Here we note the quadratic dependence on the cutoff, which is all we need in the future. Also, the distinction between $\Lambda_{0}$ and $\Lambda_{1}$ will not play any role in our subsequent discussion. We will simplify writing by replacing $\Lambda_{0,1}$ by a generic cutoff $\Lambda$ from now on.

Next we turn our attention to $Z_{q}^{(1)}$, which is the term corresponding to $n=1$ in the series in Eq.(37). (The term $n=0$ vanishes). The corresponding graph is Fig.3 with two external lines. After evaluating this graph in the presence of a constant background $\phi_{0}$, we expand to second order in the external momentum $k$, and then set $k=0$. This provides the two derivatives acting on the two external $\chi$ fields. We can then replace the two $\chi$ 's by $\phi$ 's, since these two differ only by a constant. Furthermore, repeating the argument we have used in the case of $V_{q}$, after the graph has been evaluated, we can replace $\phi_{0}$ by $\phi(\sigma, \tau)$. The result, which was computed in references [5-7], has a logarithmic dependence on the cutoff:

$$
\int_{0}^{p^{+}} d \sigma \int d \tau\left(Z_{q}^{1} \partial^{2} \phi\right)=\frac{D}{64 \pi} \ln (\Lambda / \mu) \int_{0}^{p^{+}} d \sigma \int d \tau \phi^{-5 / 2}\left(\phi\left(\partial_{\tau} \phi\right)^{2}+\left(\partial_{\sigma} \phi\right)^{2}\right) .
$$

A few comments are in order:

a) This expression involves the factor $\phi^{-5 / 2}$, which only makes sense if we agree to expand it around a constant background $\phi_{0}$. In the last section, we have identified $\phi_{0}$ with the ground state expectation value of $\phi$, and determined it by minimizing $U\left(\phi_{0}\right)$. Although, as we have seen in section 4 , the particular value of $\phi_{0}$ is somewhat dependent on the details how the model is regulated, the important point is that it does not vanish. Since, from Eqs.(18) and (19),

$$
\alpha^{\prime 2} \rightarrow \phi_{0} / \pi^{2}
$$

a non-zero $\phi_{0}$ means a non-zero string slope. We also note that a non-zero $\phi_{0}$, in addition to a non-zero slope, generates a kinetic energy term for $\phi$, and $\phi$, which was originally an auxilliary field, becomes dynamical. In what follows, so long as it is non-zero, the particular value of $\phi_{0}$ is really not important.

b) $\mu$ is an infrared cutoff, needed to avoid infrared divergence. Since, so far, we were only interested in the dependence on the ultraviolet cutoff, the infrared cutoff did not matter. Later, when we reexamine the problem from 
the point of view of the renormalization group, the basic idea will be to lower the ultraviolet cutoff $\Lambda$ to a lower value, and $\mu$ will be identified with this lower cutoff.

c) By dimensional reasoning, it is easy to show that terms corresponding to $s>1$ in the expansion (37) go with inverse powers of cutoff:

$$
Z_{q}^{(s)} \sim \Lambda^{2-2 s}
$$

Therefore, one may suspect that these terms can be ignored. Actually, this is misleading; the power suppression is compensated by the higher number of derivatives. Later on, we will see that that these terms are suppressed not by powers but by factors logarithmic in $\Lambda$.

d) We note that $s=1$ is a special case. That is the only term in the expansion that has a logarithmic dependence on the cutoff (see Eq.(40)):

$$
Z_{q}^{(1)} \sim \ln (\Lambda / \mu)
$$

So far, we have been studying $\tilde{S}_{q}$, the contribution of the field $\mathbf{q}$ to the action. To this must be added the contribution of the fermionic sector, represented by $\tilde{S}^{\prime}$ and $\tilde{S}_{f}$ in Eq.(17) to arrive at the total action. After integrating over the auxilliary fields $\kappa, \psi$ and $\bar{\psi}$, the resulting effective action, $S_{e}$, which now depends only on $\phi$, can be expanded in powers of spatial derivatives just as in Eq.(37):

$$
S_{e}=\int_{0}^{p^{+}} d \sigma \int d \tau\left(U(\phi)+\sum_{s=1}^{\infty} Z^{(s)}(\phi) \partial^{2 s} \phi\right) .
$$

We have already computed $U(\phi)$; it is essentially given by the right hand side of Eq.(29):

$$
U=\frac{\Lambda^{2}}{\pi^{2}}\left(F\left(\phi, m^{2}\right)-\frac{1}{m^{2}} \sqrt{x-x^{2}}\right)
$$

where $x$ is the solution to (see Eq.(25))

$$
\phi=\frac{x^{2}}{\bar{\alpha}^{2} m^{4}(1-x)} .
$$

Note that just as $U_{q}, U$ is quadratic in the cutoff $\Lambda$. The cutoff dependences of $Z^{(s)}$ for $s>1$ are also unchanged; they are given by simple power counting, as in Eq.(41). Also, a simple calculation shows that there is no contribution 
to $Z^{(1)}$ from the fermionic sector. To sum up:

a) The terms in Eq.(43) have the same cutoff dependence as in Eqs.(41,42): $U$ is proportional to $\Lambda^{2}$, and $Z^{(s)}$ is proportional to $\Lambda^{2-2 s}$ for $s>1$.

b) $Z^{(1)}=Z_{q}^{(1)}$ is again given by Eq.(40); and so its cutoff dependence is logarithmic.

\section{Transforming The Action}

In this section, we will try to address the following question: How does one work with an action of the form given by Eq.(43), which is both explicitly cutoff dependent, and also formally non-renormalizable, since it contains terms with more than two derivatives? Of course, here we have a somewhat unusual situation; the effective action $S_{e}$ was gotten by doing a one loop calculation, so one might think that the cutoff dependence could be eliminated by introducing counter terms. These counter terms usually arise from adding cutoff dependent pieces to the adjustable parameters (masses, coupling constants) in the theory. The adjustable parameters in our problem are $\alpha$ and $g$, and their dependence on the cutoff was already fixed by Eq.(15). We recall that Eq.(15) was forced on us by requiring the invariance of the original action under the scaling of the world sheet coordinates. We have also stressed that scaling invariance follows from invariance under a special boost, and it cannot be violated without breaking Lorentz invariance. Since there is no freedom of tuning the cutoff dependence of $\alpha$ and $g$, one might consider introducing $\phi$ dependent counter terms into $S_{e}$ to cancel, for example, the cutoff dependent part of $U$. One must remember, however, that $\phi$ (or $\beta^{2}$ ) was a non-dynamical auxilliary field in the original action of Eq.(10), and the dependence of the action on $\phi$ came from $\Delta S$ and it was completely fixed. It has later acquired a kinetic energy term (Eq.(40)) from quantum effects and as a result became dynamical. Therefore, given the initial action, the $\phi$ dependence of $S_{e}$ is completely fixed. Towards the end of this section, we will show that by suitably scaling both the world sheet coordinates and the field $\phi$, the action can be transformed into a form that is a suitable starting point for a perturbation expansion.

A second problem is how to identify the set of observable Greens functions required to be cutoff independent. We will make the reasonable assumption that physics lies in the Lorentz invariant sector of the model, and therefore we should only demand that Lorentz invariant Greens functions should be free of the cutoff. We recall that, in contrast to the covariant approach to field 
theory, we do not here have manifest Lorentz invariance. In addition, the cutoffs $a$ and $a^{\prime}$ (or $\Lambda_{0,1}$ ) are not Lorentz scalars. In view of this, it is too much to demand that all Greens functions be finite: The cutoff dependendence may be an artifact due to non-covariance, and it may disappear from the Lorentz invariant sector of the model.

This brings up the question of how to identify Lorentz invariant objects. Investigation of full Lorentz invariance is a difficult task which will not be attempted in this paper. Instead, we will construct Greens functions invariant under a subgoup of the Lorentz group consisting of rotations in the transverse directions and boost along the special light cone direction. As we have stressed earlier, the special boost is realized as scaling of the world sheet coordinates, and so we should be looking for scale invariant Greens functions. These are easy to construct, for example, the composite fields

$$
\int_{0}^{p^{+}} d \sigma \int d \tau G(\phi)(\partial \phi)^{2}, \quad \int_{0}^{p^{+}} d \sigma \int d \tau E(\phi) \partial \mathbf{q} \cdot \partial \mathbf{q}
$$

where $G$ and $E$ are arbitrary functions and $\partial$ is derivative with respect to $\sigma$ or $\tau$, are scale invariant. The Greens functions constructed from the products of these composite fields will also share this property. It is easy to construct many more examples. Note that these examples are all non-local: Since the world sheet coordinates transform under scaling, one has to integrate over them to form scale invariant quantities.

We will now argue that scale invariant objects are also cutoff independent, at least if we focus on power dependence and ignore logarithmic corrections. This follows because the only parameter in the problem that scales nontrivially is the cutoff $\Lambda$; under the scaling

$$
\sigma \rightarrow u \sigma, \tau \rightarrow u \tau
$$

$\Lambda$ transforms as

$$
\Lambda \rightarrow \Lambda / u \text {. }
$$

Therefore, the scaling dimension of any dyanamical variable must solely come from its dependence on the cutoff. For example, in the derivative expansion of Eq.(43), the cutoff dependences of $U$ and $Z^{(s)}$, given by Eq.(41), all follow from their scaling dimensions. As a special case, scale invariance implies cutoff independence.

The above discussion is incomplete in two important respects: First, it was completely classical; for example, we ignored the logarithmic dependence 
of $Z^{(1)}$, which is purely quantum in origin. Also, we have so far only considered the contributions coming from integrating over $\mathbf{q}$; we should also take into account the contributions coming from integrating over $\phi$, since the latter has become a full fledged dynamical field after the $\mathbf{q}$ integration. In the next section, we will take care of these omissions by considering a perturbation expansion, starting with $S_{e}$. The expansion parameter will initially be $1 / D$, the natural small parameter in the large $D$ limit, although it will be replaced by a more appropriate one later on. In preparation for the perturbation expansion, it is convenient to transform the action so that the free part (mass and kinetic energy) does not depend on the cutoff or on $D$. We first write $\phi$ as the sum of its expactation value $\phi_{0}$ and a fluctuating field which we called $\chi$ in Eq.(38). In order not to complicate the notation, instead of introducing a new field $\chi$, we will simply make the replacement

$$
\phi \rightarrow \phi_{0}+\phi
$$

After this replacement, we will rescale $\phi$ by

$$
\bar{\phi}=(D \ln (\Lambda / \mu))^{1 / 2} \phi
$$

and combine it with a change of the world sheet coordinates from $\sigma, \tau$ to $\bar{\sigma}, \bar{\tau}$ :

$$
\bar{\sigma}=\Lambda(\ln (\Lambda / \mu))^{-1 / 2} \sigma, \quad \bar{\tau}=\Lambda(\ln (\Lambda / \mu))^{-1 / 2} \tau .
$$

After these transformations, $S_{e}$ can be written as

$$
S_{e}=\int d \bar{\sigma} \int d \bar{\tau}\left(\bar{U}(\bar{\phi})+\sum_{1}^{\infty} \bar{Z}^{(s)}(\bar{\phi}) \partial^{2 s} \bar{\phi}\right),
$$

where the barred and unbarred quantities are related by

$$
\bar{U}=\Lambda^{-2} \ln (\Lambda / \mu) U, \quad \bar{Z}^{(s)}=\Lambda^{2 s-2}(\ln (\Lambda / \mu))^{1-s} Z^{(s)}
$$

To see more clearly what this transformation has accomplished, it is useful to expand the barred quantities in powers $\bar{\phi}$ by

$$
\bar{U}=\sum_{2}^{\infty} \bar{U}_{n} \bar{\phi}^{n}, \quad \bar{Z}^{(s)}=\sum_{1}^{\infty} \bar{Z}_{n}^{(s)} \bar{\phi}^{n},
$$

and check the dependence of these terms on the cutoff and on $D$. Defining

$$
t=\ln (\Lambda / \mu)
$$


and combining the cutoff dependence given by Eqs.(41) and (42) with the transformations described above, we see that

$$
\bar{U}_{n} \sim(D t)^{1-n / 2}
$$

and,

$$
\bar{Z}_{n}^{(1)} \sim(D t)^{(1-n) / 2}
$$

for $s=1$ and

$$
\bar{Z}_{n}^{(s)} \sim(D t)^{(1-n) / 2} t^{-s}
$$

for $s>1$. The reason for a different dependence on $t$ for $s=1$ can be traced back to $Z^{(1)}$, which is the only term in the expansion proportional to $t$.

From the above results, we see that:

a) There is no longer any dependence on powers of $\Lambda$ in the new action. The remaining cutoff dependence is purely logarithmic. One can directly see from Eqs.(51-54) that the old cutoff $\Lambda$ has been replaced by the new cutoff $t$.

b) The free part of the action, given by

$$
S_{e}^{(0)}=\int_{0}^{p^{+}} d \bar{\sigma} \int d \bar{\tau}\left(\bar{U}_{2} \bar{\phi}^{2}+\bar{Z}_{1}^{1} \bar{\phi} \partial^{2} \bar{\phi}\right)
$$

is both cutoff and $D$ independent. We recall that this was the main motivation for transforming the action. Identifying $D t$ with the wave function renormalization constant, we note that the scaling of $\phi$ in Eq.(46) very much looks like the standard wave function renormalization of field theory. There is, however, an important difference: Wave function renormalization constant normally looks like

$$
Z \sim 1+e^{2} \ln (\Lambda / \mu)
$$

whereas, with the identification $e^{2}=1 / D$, we have

$$
Z \sim e^{-2} \ln (\Lambda / \mu)
$$

This is because, in our case, the kinetic energy term for $\phi$ is not present in the original action, but it is generated at the level of one loop.

c) Examining Eqs. $(52,53)$, we see that, instead of $1 / \sqrt{D}$, it is more natural to identify

$$
\lambda=1 / \sqrt{D t}
$$

as the parameter of the perturbation expansion. This can be made more precise by defining $\lambda$ by setting the coefficient of the term with $s=1, n=2$ 
equal to $\lambda$, so that, up to an irrelevant multiplicative constant, it appears in the action in the form

$$
\int d \sigma \int d \tau\left(\lambda \bar{\phi}^{2} \partial^{2} \bar{\phi}\right)
$$

With this new identification of the coupling constant, the convergence of the expansion around the meanfield is no longer tied to the number of transverse dimensions being large: $\lambda$ could be small without $D$ being large if $\Lambda / \mu$ is large. Later on, we will see that one can identify $\lambda$ as the running coupling constant defined at scale $\mu$. As $\mu \rightarrow 0$, the model tends towards a free field theory. There are, however, obstacles in the infrared that prevent us from reaching $\mu=0$. For one thing, we have decompactified the sigma coordinate and replaced a discrete spectrum by a continuous one. This approximation, valid in the ultraviolet region, breaks down when we approach the infrared. In addition, there is a problem with a strongly coupled zero mode in the spectrum [11]. Also, by just dimensional reasoning, the $\mu$ dependence of the $Z$ 's is given by

$$
Z^{(s)} \sim \mu^{2 s-2}
$$

for $s>1$, so there is a serious infrared divergence. All we can say is that, as we reduce $\mu$, at least for a while, $\lambda$ tends to decrease. We will make this point a bit more precise when we discuss the renormalization group.

Now we are ready to give at least a partial answer to the question posed at the beginning of this section. We have constructed a new action, Eq.(48), in which the original cutoff $\Lambda$ is replaced by the logarithmic cutoff $t$. For computing Lorentz and hence scale invariant quantities, it can be used in place of the old action of Eq.(43), since the two are related by a scale transformation. The free part of the action is cutoff independent, and the only cutoff dependence appears in the interaction. If formally the limit $t \rightarrow \infty$ is taken, the theory becomes free. Of course, one still have to deal with the additional cutoff dependence coming from higher order terms, but at least, there is now a satisfactory starting point, eq.(48), for doing the perturbation expansion.

\section{Perturbation Expansion And The Renormalization Group}

Our first goal is to determine the dependence of the individual terms of the perturbation expansion on the cutoff $t$ for large $t$. Later we will use the knowledge thus gained to introduce an approach to the problem based on the renormalization group. We have already noted that, for large $t$, the 
expansion parameter $\lambda$ is small, and so the perturbative treatment is justified. The tool we will use to investigate the large $t$ dependence is elementary power counting. Let us first introduce a uniform notation for interaction vertices by defining

$$
W_{n}^{(0)}=\bar{U}_{n}, \quad W_{n}^{(s)}=\bar{Z}_{n+1}^{(s)}, \quad s \geq 1, n>2 .
$$

The subscript $n$ on $W$ counts the number of lines meeting at the vertex, and $2 s$ counts the total number of derivatives (momenta) associated with various lines. The asymptotic $t$ dependence of the $W$ 's can be read from Eqs.(52-54):

$$
W_{n}^{(s)} \sim t^{\left(1-s-n / 2+\delta_{1, s}\right)} .
$$

Now consider a contribution to the vertex $W_{E}^{(S)}$ from a graph with $E$ external lines, $I$ internal lines, $V$ vertices and $L$ loops (unconstrained momenta). Very schematically, this graph can be represented as

$$
W_{E}^{(S)} \sim \int^{\sqrt{t}} \prod_{i=1}^{L} d^{2} k_{i} \prod_{j=1}^{I}\left(k_{j}^{2}+M^{2}\right)^{-1} \prod_{l, m=1}^{V}\left(\left(k_{l}\right)^{2 s_{l}-2 s_{m}} W_{n_{l}}^{\left(s_{l}\right)}\right),
$$

where, $n_{l}$ represents the number of lines entering the $l$ 'th vertex and $2 s_{l}$ the number of momenta associated with that vertex. Of these momenta, $2 s_{m}$ of them are associated with external lines and the rest with internal lines. These quantities satisfy the following constraints:

$$
\sum_{l=1}^{V} n_{l}=E+2 I, \quad \sum_{m=1}^{V} s_{m}=S, \quad V=I-L+1 .
$$

In writing Eq.(59), we have suppressed many details; for example, we have not expressed $k_{l}$ 's, the momenta entering the vertices, as linear combinations of the internal momenta $k_{i}$. Also, Lorentz indices have been suppressed. Since we are only interested in counting powers of $t$, these details do not matter.

To find the large $t$ behaviour of $W_{E}^{(S)}$, we use Eq.(58) for the $W_{n}^{(s)}$ 's on the right side, and also, we change variables by

$$
k_{i} \rightarrow \sqrt{t} k_{i} .
$$

The result is

$$
W_{E}^{(S)} \sim t^{L-I} \prod_{l, m=1}^{V} t^{1-s_{m}-n_{l} / 2+\delta_{s_{l}, 1}}=t^{L-2 I-S-E / 2} \prod_{l=1}^{V} t^{1+\delta_{s_{l}, 1}},
$$


where (60) was used in the second step. Clearly, the dominant contribution comes from $s_{l}=1$, corresponding to vertices $W_{n}^{(1)}$. Letting $s_{l}=1$ in the above expression, and using $V=I-L+1$, we have,

$$
W_{E}^{(S)} \sim t^{L-2 I-S+2 V-E / 2}=t^{-L-S-E / 2+2} .
$$

We see that one loop contribution, $L=1$ dominates, and finally, the leading $t$ dependence is

$$
W_{E}^{(S)} \sim t^{1-S-E / 2}
$$

But this is just this the original $t$ dependence given by (58) for vertices with $S>1$. Therefore, higher order contributions do not change the power dependence of vertices with $S>1$, although they do contribute to the constant multiplying this power. In the case of vertices with $S=1$, the power given by (63) is down by one unit compared to the original one (Eq.(58)), so in this case there is no correction to the original dominant asymptotic behaviour. To summarize:

a) For large $t$, the theory is weakly coupled, with the coupling constant $\lambda$ given by Eq.(56).

b) The $t$ dependences of the vertices given by Eq.(58) are unchanged when higher order perturbation results are taken into account.

c) Perturbatively, the vertices $W_{n}^{(1)}$, with $s=1$, dominate the large $t$ behaviour; $W$ 's with $s>1$ are negligible in comparison. We note that these are the so called non-renormalizable interaction terms with more than two derivatives. In fact, they are damped by inverse powers of $t$ and are therefore harmless.

d) The $W$ 's with $s=1$ themselves receive no higher order corrections. These vertices are therefore still given by Eq.(40): One has to expand the right hand side of this equation in powers of $\phi$ about a non-zero field expectation value.

We will now reexamine the results obtained so far from the point of view of the renormalization group. The initial model was regulated by a grid on the world sheet. The grid spacing, or equivalently, a corresponding momentum cutoff $\Lambda$ acted as the regulator. The basic idea of the renormalization group is to lower the cutoff systematically by integrating over slices of momenta, finally arriving at an effective action sensitive only to low values of momenta (energy). Here, we can implement this idea as follows: We first start with a very large cutoff $\Lambda^{(0)}$, and integrate over the modes between $\Lambda^{(0)}$ and $\Lambda$ of the field $\mathbf{q}$, where the ratio $\Lambda^{(0)} / \Lambda$ is taken to be large. The result of this 
integration can be schemetically represented by

$$
S\left(\Lambda_{0}\right)=S_{q}(\Lambda)+S_{e}\left(\Lambda^{(0)}, \Lambda\right)
$$

In this equation, $S\left(\Lambda^{(0)}\right)$ stands for the original action, with the modes of the field q cutoff at $\Lambda^{(0)}$. After integrating over the modes between $\Lambda^{(0)}$ and $\Lambda$, and carrying out the large $D$ expansion, as we have done in sections 3 to 5 , we end up with the two terms on the right hand side. The first term,

$$
S_{q}(\Lambda)=\int d \sigma \int d \tau\left(\frac{1}{2} \mathbf{q}^{\prime 2}+\frac{1}{2}\left(\phi_{0}+\lambda \bar{\phi}\right) \dot{\mathbf{q}}^{2}\right) E(\Lambda),
$$

is the original action for $\mathbf{q}$, with, however, the cutoff reduced from $\Lambda^{(0)}$ to $\Lambda$. Here $E(\Lambda)$ is a projection operator which cuts off the modes of both $\mathbf{q}$ and $\phi$ at $\Lambda$. Compared to Eq.(36), we have $\phi_{0}+\lambda \bar{\phi}$ in place of simply $\phi$ because of the shift $\phi \rightarrow \phi_{0}+\phi$ (Eq.(45)), followed by the scaling $\phi=\lambda \bar{\phi}$ (Eq.(46)).

The second term on the right side of the equation is the action for $\phi$, generated by the integration over the modes of $\mathbf{q}$ between $\Lambda^{(0)}$ and $\Lambda$. This term can again be expanded as in Eq.(43). The only difference is in the cutoffs used in computing it: The ultraviolet cutoff is now $\Lambda^{(0)}$ and the infrared cutoff is $\Lambda$. If the transformation to the barred variables is carried out, everything works out the same as before, with the exception that, $t$ is now replaced by

$$
t \rightarrow \ln \left(\Lambda^{(0)} / \Lambda\right)
$$

The reason for starting with an initial cutoff $\Lambda^{(0)}$ instead of immediately with $\Lambda$ is the following: The field $\phi$ did not have a kinetic energy term in the initial action, and such a term is needed in order to be able to integrate perturbatively over this field. Integrating only over the modes of $\mathbf{q}$ from $\Lambda^{(0)}$ to $\Lambda$ initially generates the needed kinetic energy term. The ratio $\Lambda^{(0)} / \Lambda$ is taken to be large so that the coupling constant is small and therefore perturbation expansion is valid. Once the kinetic energy term for $\phi$ is generated, one can take $S\left(\Lambda^{(0)}\right)$ as the starting point of the renormalization group analysis. Having reduced the cutoff from $\Lambda^{(0)}$ to $\Lambda, \Lambda$ becomes the new ultraviolet cutoff. We can continue reducing $\Lambda$ further by integrating over the modes of both $\mathbf{q}$ and $\phi$ together till we reach a much lower scale $\mu$. Following the conventional treatment, this can be done in infinitesimal steps, and a set of coupled differential equations for the $W$ 's can be derived. However, here the situation is much simpler; we can dispense with this elaborate machinery, relying instead on the results from perturbation theory, summarized following 
Eq.(63). As we keep on reducing $\Lambda$ by integrating over the modes of $\mathbf{q}$ and $\bar{\phi}$, the coupling constant $\lambda$ is still given by Eq.(56), with the only difference that the definition of $t$ keeps changing. If $\Lambda$ is reduced to a new value $\mu$, the corresponding $t$ is given by

$$
t \rightarrow \ln \left(\Lambda^{(0)} / \mu\right)
$$

This is because to the leading order in $t, \lambda$ receives no contribution from the integration over the modes $\bar{\phi}$ (see item $d$ ) of the summary following Eq.(63)). The only contribution comes from the integration over the modes of $\mathbf{q}$, and that simply changes the lower cutoff in Eq.(51) from $\Lambda$ to $\mu$. As a consequence, the coupling constant runs towards zero as the lower cutoff tends to zero, and the model appears to flow towards a free theory in the infrared. Of course, we pointed out at the end of section 6 , as $\mu$ gets small, at some point the approximations we are making are no longer valid. It is very likely that at some value of $\mu$, the coupling constant $\lambda$ reaches a minimum, and as $\mu$ is decreased further, it starts growing. The theory then becomes strongly coupled, and the mean field method, based on the expansion in $\lambda$, breaks down.

All this is telling us that the mean field approximation is good when applied to highly excited states of the string, but it breaks down when applied to the lower lying spectrum. One can therefore trust it for computing the asymptotic slope, but not for computing, for example, the intercept. To see this more clearly, instead of letting $\mu \rightarrow 0$, we can let $\Lambda \rightarrow \infty$, while still keeping $\mu$ large. In this limit, $\lambda$ again tends to zero, the fluctuations of the string slope tend to die out, and to a good approximation, we get a free string with a fixed slope. We note that in the light cone variables we have chosen, $\mu p^{+}$corresponds to the square of the total momentum. Therefore, at large $\mu$, we are probing the higher excitations of the string, with the square of the mass of the order of $\mu p^{+}$or larger. It is only in this regime that we can trust the perturbation expansion, and the resulting picture of formation of a free string.

\section{Conclusions And Open Problems}

In the present paper, we continued earlier work on the world sheet approach to field theory and the mean field calculations based on it. In particular, we focused on the cutoffs needed for the world sheet set up, and we investigated possible cutoff dependences of various results based on the mean 
field method. We showed that after integrating over the target space field q, a new field $\phi$ was dynamically generated, with a generalized sigma model for its action. This action was explicitly cutoff dependent; however, after a simple transformation, all the cutoff dependence could be absorbed into the definition of a running coupling constant. An argument based on the idea of the renormalization group was used to show that, as the cutoff was lowered, the coupling constant approached zero, and the model tended towards a free string. We argued, however, that because of various infrared problems, it was not possible to really reach this limit. Nevertheless, by keeping the cutoff sufficiently large, we could still obtain useful information about the ultraviolet behaviour of the model: For example, one could conclude that the higher exited modes were indeed described by a free string. We consider this as the main result of the present work.

Many problems, some of them listed at the beginning of the introduction, such as full Lorentz invariance, still remain open. Another problem, brought to light here, is the likely breakdown of the mean field expansion for the low lying states of the string. It would be very desirable to find a way of extending the mean field method, so that, for example, the string intercept could be reliably determined.

\section{Acknowledgement}

This work was supported in part by the Director, Office of Science, Office of High Energy and Nuclear Physics, of the U.S. Department of Energy under Contract DE-AC02-05CH11231.

\section{References}

1. K.Bardakci, C.B.Thorn, Nucl.Phys. B 626 (2002) 287, hep-th/0110301.

2. K.Bardakci, C.B.Thorn, Nucl.Phys. B 652 (2003) 196, hep-th/0206205.

3. K.Bardakci, C.B.Thorn, Nucl.Phys. B 661 (2003) 235, hep-th/0212254.

4. K.Bardakci, Nucl.Phys. B 667 (2004) 354, hep-th/0308197.

5. K.Bardakci, Nucl.Phys. B 698 (2004) 202, hep-th/0404076.

6. K.Bardakci, Nucl.Phys. B 715 (2005) 141, hep-th/0501107.

7. K.Bardakci, Nucl.Phys. B 746 (2006) 136, hep-th/0602131. 
8. For some alternative approaches on putting field theory on the world sheet, see O.Aharony, Z.Komargodski, S.S.Razamat, JHEP 005 (2006) 016, hep-th/0602226, J.R.David, R.Gopakumar, From Spacetime to Worldsheet: Four Point Correlators, hep-th/0606078, A.Clark, A.Karch, P.Kovtun, D.Yamada, Phys.Rev. D 68 (2003) 066011, hep-th/0304107.

9. For an investigation of renormalization and Lorentz invariance in the light cone formulation, see C.B.Thorn, Nucl.Phys. B 699 (2004) 427, hep-th/0405018.

10. G.'t Hooft, Nucl.Phys. B 72 (1974) 461.

11. For a review of the large N method, see M.Moshe, J.Zinn-Justin, Phys.Rep.385 (2003) 69, hep-th/0306133. 